\title{
Development and Validation of an LC-MS/MS Method for Determination of $p$-Phenylenediamine and its Metabolites in Blood Samples
}

\author{
Khaled M. Mohamed ${ }^{1}$, Duncan Cromarty $^{2}$ and Vanessa Steenkamp ${ }^{2}$ \\ ${ }^{1}$ Assiut Chemical Laboratory, Medico-Legal Department, Ministry of Justice, Assiut, \\ Egypt \\ ${ }^{2}$ Department of Pharmacology, Faculty of Health Sciences, University of Pretoria, \\ Pretoria, South Africa
}

Corresponding author contact: khaled.masoud@yahoo.com, Tel: 00201003100517, Fax: 0020882324038

\begin{abstract}
In some developing countries, $p$-phenylenediamine (PPD) is used in combination with Henna as hair dye or skin decoration. A sensitive LC-MS/MS method was developed and validated for the simultaneous determination of $p$-phenylenediamine (PPD) and its metabolites $N$-acetyl-p-phenylenediamine (MAPPD) and $N, N$-diacetyl- $p$ phenylenediamine (DAPPD) in human blood. Acetanilide was used as an internal standard (IS). The LC-MS/MS was operated under multiple reaction-monitoring mode using the electrospray positive ionization technique. The transition ions $\mathrm{m} / \mathrm{z} 109 \rightarrow$ $92, \mathrm{~m} / \mathrm{z} 151 \rightarrow 92, \mathrm{~m} / \mathrm{z} 193 \rightarrow 92$, and $\mathrm{m} / \mathrm{z} 136 \rightarrow 77$ were selected for the quantification of PPD, MAPPD, DAPPD, and IS, respectively. The linear range was 10-2000 ng/mL for all the compounds. The absolute recoveries were 51.94, 56.20 and $54.88 \%$ for PPD, MAPPD and DAPPD, respectively. Intra- and inter-assay imprecision were lower than 14\% (RSD), and the bias of the assay was lower than $15 \%$ for all the compounds. The stability studies demonstrated that critical degradation for PPD in blood samples and autosampler occurred after $6 \mathrm{~h}$, while MAPPD and DAPPD were stable in blood samples and the autosampler up to $48 \mathrm{~h}$ and $24 \mathrm{~h}$, respectively. This newly developed method allows for the detection of PPD and its metabolites in blood samples in the clinical and forensic setting.
\end{abstract}

Keywords: $p$-Phenylenediamine; Metabolites; Blood; LC-MS/MS; Validation; Stability 


\section{Introduction}

$p$-phenylenediamine (PPD) is an azo dye intermediate used for dying furs, photochemical measurements, as a photographic developing agent, as an intermediate in manufacture of antioxidants and as accelerators for rubbers. In some African and Asian countries, this chemical is used alone or in combination with Henna for dyeing of hair and skin [1-3]. A vast numbers of suicidal, homicidal and accidental poisoning cases involving PPD have been recorded [4-7].

In 20 fatal p-phenylenediamine poisoning cases, convulsion, facial edema and cyanosis were characteristic whereas edemas of the epiglottis and vocal folds were observed in all cases [8] change number. Other poisoning symptoms after oral intake include vomiting, epigastralgia, edema of the neck and pharynx, dyspnea, acute renal failure, rhabdomyolysis, hemolysis, methemoglobinemia and hepatic failure [8-10]. PPD is rapidly absorbed into blood through mucous membranes of the digestive tract after its oral intake, and metabolized into quinonediimine, which acts as a cytotoxin. It is acetylated into $N$-acetyl-p-phenylenediamine (MAPPD) and $N, N$-diacetylpphenylenediamine (DAPPD) as the major metabolites for detoxification to be excreted into urine [11-13].

PPD has been detected in blood, urine and gastric content by gas chromatographymass spectrometry (GC-MS) after liquid-liquid extraction [14,15]. Methods involving liquid chromatography (LC) with electrochemical detector [16], ultraviolet [16,17], diode array [18], or MALDI-MS/MS [17] for quantification of PPD and its metabolites have been reported. LC-MS/MS is currently the classical analytical tool in forensic and clinical laboratories for the analysis of most of the common drugs and toxic substances in biological matrices.

A validated LC-MS/MS method for detection and quantification of PPD and its metabolites in human blood has not been described to date. Therefore, the aim of this study was to a sensitive LC-MS/MS method for the simultaneous determination of PPD, MAPPD and DAPPD in human blood. The method was successfully applied to analysis of human blood samples collected from postmortem cases provided by Assiut Forensic Chemistry Laboratory of Medico-Legal Department, Ministry of Justice, Egypt. 


\section{Materials and methods}

\subsection{Reagents and standards}

p-Phenylenediamine dihydrochloride (99\%), $N$-acetyl-p-phenylenediamine (99\%), tert.-Butyl methyl ether (99.8) and acetanilide (99\%) were purchased from SigmaAldrich. N,N-diacetyl-p-phenylenediamine (DAPPD) was synthesized in our laboratory [13]. Formic acid (98\%), ammonium acetate (98\%) and ammonium formate (99\%) were purchased from Fluka Chemie GmbH. Acetonitrile (99.9\%) and ammonium hydroxide (25\%) were purchased from Merck. Methylene chloride (HPLC) and Ethly acetate (HPLC) were purchased from Baxter. Methanol (99.9\%) was purchased from Romil pure Chemistry.

\subsection{Instrumentation}

An Agilent LC 1100 binary pump, autosampler, vacuum degasser, column oven (Agilent Technologies), and a Eclipse XDB C18 column (150 mm x $4.6 \mathrm{~mm}, 5 \mu)$ were carried out for chromatographic separations. The mass spectrometric analysis was performed by use of an AB/MDS Sciex 4000 QTrap LC-MS/MS (Applied Biosystems, Canada) instrument in triple quadrupole mode, equipped with an AB/MDS Sciex Turbo Ion Spray interface. The software was Analyst 1.4.1.

\subsection{Liquid chromatography}

The analytical column was maintained at $25{ }^{\circ} \mathrm{C}$. A mobile phase gradient pumped at 1 $\mathrm{mL} / \mathrm{min}$ was used to elute the analytes from the column. Mobile phase A consisted of acetonitrile. Mobile phase B consisted of $0.1 \%$ formic acid. The gradient was initiated at $100 \% \mathrm{~B}$ for $2 \mathrm{~min}$, and subsequently a linear gradient led to $70 \% \mathrm{~B}$ in $1 \mathrm{~min}$, kept for $2.75 \mathrm{~min}$ and brought back to $100 \% \mathrm{~B}$ in $0.25 \mathrm{~min}$. The column was equilibrated for $2 \mathrm{~min}$. Total run time was $8 \mathrm{~min}$. Injection volume was $10 \mu \mathrm{L}$.

\subsection{Mass spectrometry}

Detection of analytes and IS was performed on a triple quadrupole mass spectrometer operating in the positive mode $\left(\mathrm{ESI}^{+}\right)$with multiple reaction monitoring (MRM). The most abundant fragment for each compound was selected by performing enhanced product ion scans of the standards during an infusion analysis using a Harvard syringe pump at a constant flow rate of $10 \mu \mathrm{L} / \mathrm{min}$. For each compound, two or three mass 
Table 2

Summary of calibration curves for PPD, MAPPD and DAPPD in human blood $(n=3)$ with back calculated concentrations.

\begin{tabular}{|c|c|c|c|c|c|c|c|c|}
\hline & \multicolumn{6}{|c|}{ Concentration (ng/mL) } & \multicolumn{2}{|c|}{ Regression Equation } \\
\hline & 10 & 50 & 100 & 500 & 1000 & 2000 & Slope & $r^{2}$ \\
\hline \multicolumn{9}{|l|}{ PPD } \\
\hline Mean & 10.6 & 53.9 & 108.3 & 519.3 & 971.7 & 1960.0 & 0.0140 & 0.9853 \\
\hline SD & 0.3 & 3.8 & 4.0 & 35.5 & 59.7 & 113.6 & 0.0069 & 0.0127 \\
\hline$\%$ RSD & 2.9 & 7.1 & 3.7 & 6.8 & 6.1 & 5.8 & 0.4929 & 0.0129 \\
\hline$\%$ Bias & 5.7 & 7.9 & 8.3 & 3.9 & -2.8 & -2.0 & & \\
\hline \multicolumn{9}{|l|}{ MAPPD } \\
\hline Mean & 10.3 & 49.0 & 109.3 & 522.7 & 1008.3 & 2086.7 & 0.0196 & 0.9807 \\
\hline SD & 0.6 & 1.8 & 5.0 & 32.1 & 28.4 & 181.5 & 0.0060 & 0.0083 \\
\hline$\%$ RSD & 5.5 & 3.7 & 4.6 & 6.1 & 2.8 & 8.7 & 0.3049 & 0.0084 \\
\hline$\%$ Bias & 2.7 & -1.9 & 9.3 & 4.5 & 0.8 & 4.3 & & \\
\hline \multicolumn{9}{|l|}{ DAPPD } \\
\hline Mean & 11.1 & 48.7 & 110.0 & 520.7 & 1022.0 & 2060.0 & 0.0099 & 0.9897 \\
\hline SD & 0.4 & 4.1 & 6.1 & 26.1 & 87.0 & 120.0 & 0.0021 & 0.0154 \\
\hline$\%$ RSD & 3.9 & 8.4 & 5.5 & 5.0 & 8.5 & 5.8 & 0.2073 & 0.0155 \\
\hline$\%$ Bias & 11.0 & -2.6 & 10.0 & 4.1 & 2.2 & 3.0 & & \\
\hline
\end{tabular}

SD: Standard deviation

RSD: Relative standard deviation

$r^{2}$ : Correlation coefficient 

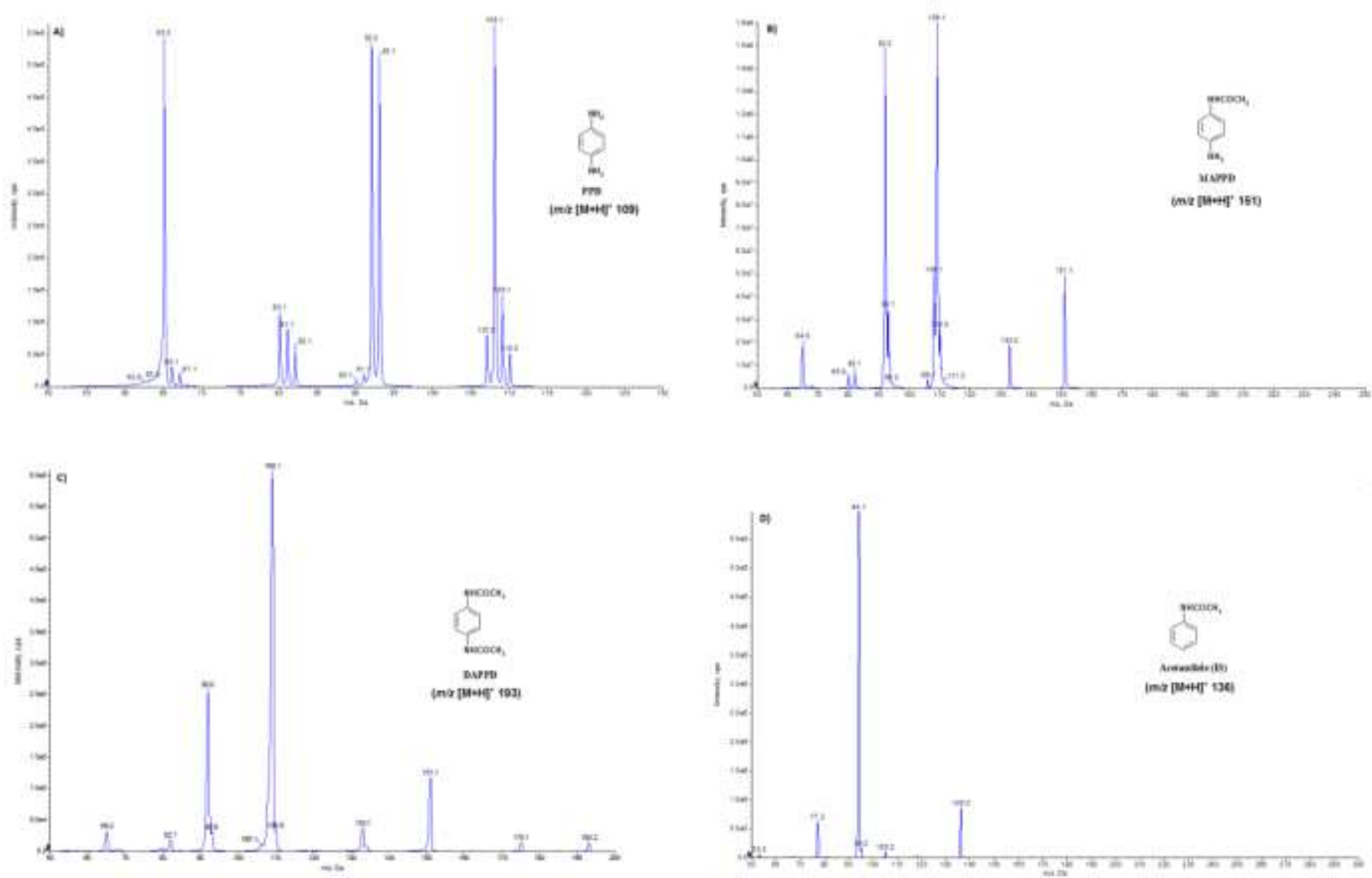

Fig 1. MS-MS spectra in the positive mode $\left(\mathrm{ESI}^{+}\right)$of the precursor ions at m/z 109 for PPD (A), m/z 151 for MAPPD (B), m/z 193 for DAPPD (C), and $\mathrm{m} / \mathrm{z} 136$ for IS (D). 
fragments were monitored with one fragment used for quantification and the other fragments used for the additional confirmation of identity (Fig. 1). The MRM transitions are reflected in Table 1. The compound dependent parameters like the collision energy (CE) and declustering potential (DP) were adjusted to provide the highest sensitivity (Table 1). Compound independent parameters that remained constant were as follows: curtain gas (CUR): 23 psi; Ion spray voltage (ISV): $5500 \mathrm{~V}$; Ion source temperature (TEM): $450^{\circ} \mathrm{C}$; Ion source gas 1 (GS1): $36 \mathrm{psi}$; Ion source gas 2 (GS2): 45 psi; Collision gas (CAD): medium; Entrance potential (EP): $10 \mathrm{~V}$; Collision cell exit potential (CXP): $10 \mathrm{~V}$; Interface heater (Ihe), on. Quadrupole 1 and quadrupole 3 were maintained at unit resolution. Dwell time set was $100 \mathrm{~ms}$ for all compounds.

\section{Table 1}

Mass spectrometry parameters.

\begin{tabular}{ccccc}
\hline Analyte & RT & MRM & CE & DP \\
& $(\mathrm{m} / \mathrm{z})$ & $(\mathrm{m} / \mathrm{z})$ & $(\mathrm{m} / \mathrm{z})$ & $(\mathrm{m} / \mathrm{z})$ \\
\hline PPD & 1.6 & $109 \rightarrow 92^{*}$ & 22 & 45 \\
& & $109 \rightarrow 65$ & 33 & 45 \\
MAPPD & 2.0 & $151 \rightarrow 133$ & 20 & 28 \\
& & $151 \rightarrow 109$ & 28 & 28 \\
& & $151 \rightarrow 92^{*}$ & 32 & 28 \\
DAPPD & 5.1 & $193 \rightarrow 151$ & 25 & 60 \\
& & $193 \rightarrow 109$ & 37 & 60 \\
& & $193 \rightarrow 92^{*}$ & 42 & 60 \\
& & $136 \rightarrow 94$ & 21 & 45 \\
& & $136 \rightarrow 77$ & 36 & 45 \\
\hline
\end{tabular}

* Transition used in the quantification. 


\subsection{Calibrators and controls}

Stock solution of PPD with a concentration of $1.0 \mathrm{mg} / \mathrm{mL}$ was prepared by dissolving $10 \mathrm{mg}$ free base of PPD-dihydrochloride in $10 \mathrm{~mL}$ water. Stock solutions of MAPPD and DAPPD $(1 \mathrm{mg} / \mathrm{mL})$ were prepared separately by dissolving $10 \mathrm{mg}$ of each analyte in $10 \mathrm{~mL}$ methanol. Working solutions were prepared by diluting the stock solutions of each analyte to a final concentration of $100 \mu \mathrm{g} / \mathrm{mL}$. Different stock standards were used to prepare quality control (QC) samples at the same concentrations. Working calibrators $(10,50,100,500,1000$ and $2000 \mathrm{ng} / \mathrm{mL})$ for PPD, MAPPD and DAPPD were made in blank blood. Low, medium and high quality controls (LQC, MQC and HQC) were also prepared in blank blood at concentration of 75, 750 and $1500 \mathrm{ng} / \mathrm{mL}$ for all analytes. Working internal standard containing $200 \mathrm{ng} / \mathrm{mL}$ of acetanilide was prepared by diluting the stock solutions of acetanilide $(1.0 \mathrm{mg} / \mathrm{mL})$ with methanol. Standard solutions were stored at $-20{ }^{\circ} \mathrm{C}$ until use.

\subsection{Sample preparation}

To $10 \mathrm{~mL}$ polypropylene tubes was added; $0.5 \mathrm{~mL}$ of blood, $100 \mu \mathrm{L}$ of $200 \mathrm{ng} / \mathrm{mL}$ acetanilide (IS), $100 \mu \mathrm{L}$ of concentrated ammonium hydroxide (33\%) and $4.0 \mathrm{~mL}$ of dichloromethane. The tubes were then vortex mixed for 5-min and centrifuged for 3 min. The organic layer was transferred to $7 \mathrm{~mL}$ glass tubes and evaporated to dryness using a speed vacuum concentrator at $35^{\circ} \mathrm{C}$. The dried extracts were reconstituted in $100 \mu \mathrm{L}$ of $1 \%$ formic acid in acetonitrile and $10 \mu \mathrm{L}$ was injected into the LC-MS/MS system.

\subsection{Validation}

The method was validated to general requirements to meet the USFDA guidelines [19]. Specificity, sensitivity, linearity, inter- and inter-assay imprecision, accuracy, recovery, matrix effect, dilution integrity and stability were assessed to evaluate method integrity.

\subsubsection{Specificity, sensitivity and linearity}

Seven different blank blood specimens (no analyte or IS) were used to evaluate the co-eluting chromatographic peaks that might interfere with detection of analytes or IS. 
The limits of detection (LOD) and quantification (LOQ) for each analyte were determined as analyte concentrations giving signal-to-noise ratio $(\mathrm{S} / \mathrm{N})$ of 3 and 10 , respectively.

Calibration graphs for PPD, MAPPD and DAPPD were established in the range of 10-2000 ng/mL blood. Calibration curves were constructed by plotting the peak area ratio of the analyte to the IS versus analyte concentration. Linearity of the method based on peak area ratios was evaluated by coefficient of determination $\left(r^{2}\right)$.

\subsubsection{Imprecision, accuracy and dilution integrity}

Intra- and inter-assay accuracy and precision for each analyte were estimated at HQC, MQC and LQC (75, 750 and $1500 \mathrm{ng} / \mathrm{mL})$ in five replicates. The accuracy and imprecision was calculated and expressed in terms of percent bias and percent relative standard deviation (\%RSD), respectively.

To investigate dilution integrity, spiked sample at concentration of $10000 \mathrm{ng} / \mathrm{mL}$ was prepared and diluted 10 times with blank blood in four replicates. Each concentration was calculated by applying the dilution factor of 10 against the freshly prepared calibration curve for PPD, MAPPD and DAPPD.

\subsubsection{Matrix effects, recovery and stability}

To evaluate the magnitude of matrix ion suppression/ enhancement effects on the MRM LC-MS/MS sensitivity, a comparison between the peak areas of extracted blank blood samples spiked with standards at QC2 concentrations after the extraction procedure and the peak areas of pure diluted standards at the same concentrations [20].

The extraction efficiency (\%) of the analytes was determined at HQC, MQC and LQC $(n=4)$. It was calculated by comparing peak areas obtained from the extracted samples to with those achieved after direct injections of standard solutions at the same concentrations.

In order to assess the stability of the analytes in human blood, two quality control samples MQC and LQC $(n=4)$ were prepared and stored at $-20{ }^{\circ} \mathrm{C}$ for $6,12,24$ and $48 \mathrm{~h}$. Concentrations were calculated and the corresponding means were compared.

Stability of analytes after extraction was evaluated over $24 \mathrm{~h}$. Extracted QCs were analyzed immediately after extraction along with calibrators, and re-injected again after 4, 6, 12 and $24 \mathrm{~h}$. 


\subsection{Application of the method}

Antemortem and postmortem blood samples were collected from poisoning cases by PPD at Assiut Chemical Laboratory, Medico-Legal Department, Ministry of Justice, and Hospital Sohag University, Egypt. This study protocol was approved by the ethics advise committee of Medico-Legal Department, Ministry of Justice. Postmortem human blood samples were stored at $-20{ }^{0} \mathrm{C}$ until analysis.

\section{Results and discussion}

\subsection{Optimization of the chromatographic conditions and extraction}

Various mixture(s) of isocratic of acetonitrile or methanol with different buffers such as ammonium acetate, ammonium formate, acetic acid and formic acid at variable $\mathrm{pH}$ range of 3.5-5.5 were tested for complete chromatographic resolution of PPD, MAPPD, DAPPD, and IS.

Also, different gradients of acetonitrile and $0.1 \%$ formic acid were assayed at a constant flow rate of $1.0 \mathrm{~mL} / \mathrm{min}$ in order to get all analytes separated. Mobile phase comprising of $0.1 \%$ formic acid:acetonitrile in gradient pumped at $1 \mathrm{~mL} / \mathrm{min}$ was found to be suitable during LC optimization. The retention times of the PPD, MAPPD, DAPPD and the internal standard (IS; acetanilide) were 1.64, 2.01, 5.09 and

\subsection{3 min, respectively}

Since PPD is a water-soluble, it was necessary in the method development to optimize extraction procedure. In this work, one-step liquid-liquid or solid phase extraction were tested to isolate PPD, MAPPD and DAPPD from blood samples. Liquid-liquid extraction was examined using different organic solvent such as dichloromethane, ethylacetate, methyl-tert-butyl and ethylacetate:hexane (1:4). A clean chromatogram and sufficient extraction recoveries for all analytes were obtained with dichloromethane. A different solid phase extraction cartridge such as Bond elut Plexa, C18 and C8 cartridges has been tested. Very poor recoveries were obtained for both PPD and MAPPD (15-20\%) with all cartridges. 

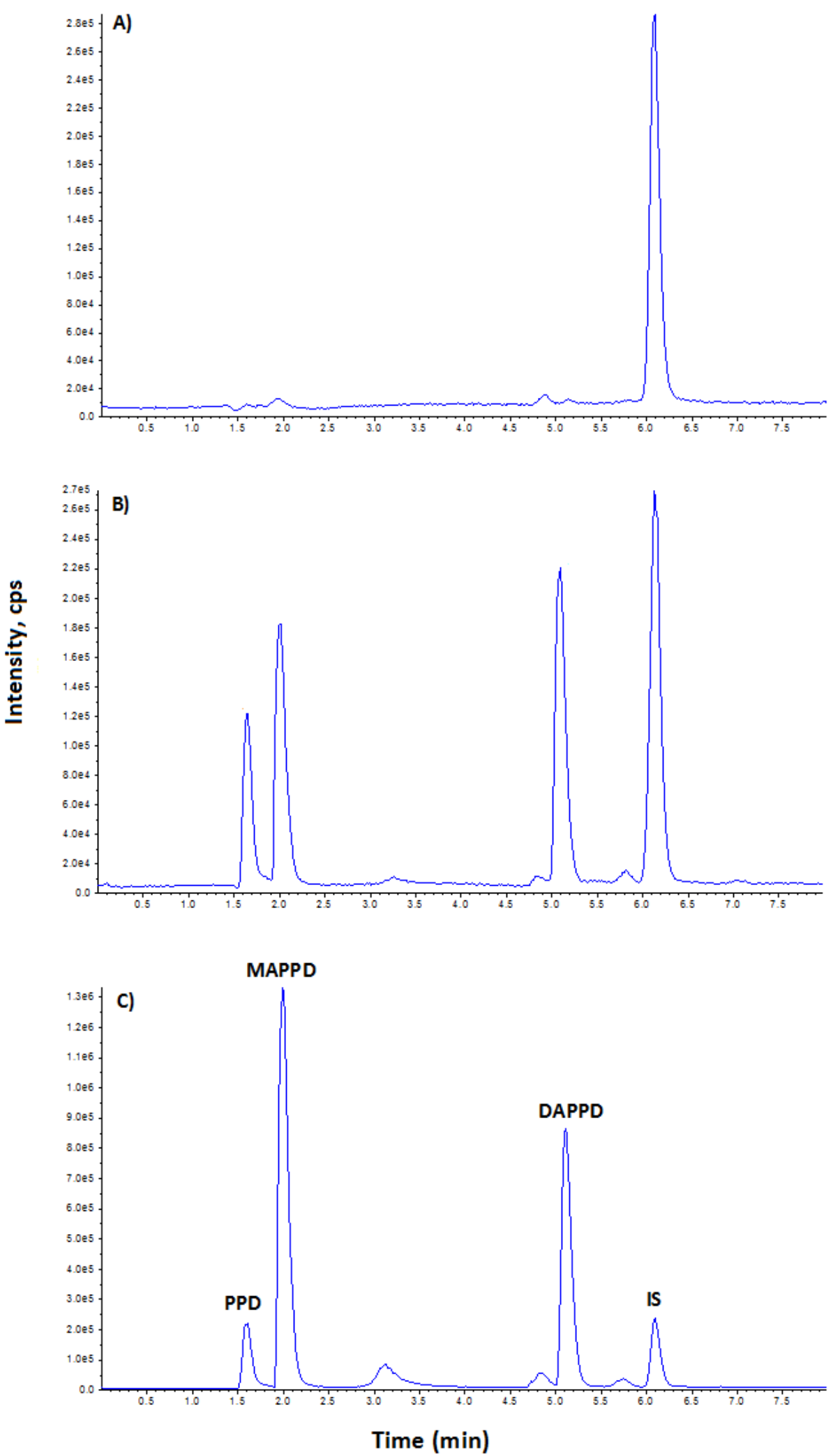

Fig 2. Total ion chromatograms (TIC) for the analysis of a blank blood with IS (A), blood sample spiked with $30 \mathrm{ng} / \mathrm{mL}$ of PPD, MAPPD and DAPPD (B) and blood sample collected from postmortem case (C). 
Table 2

Summary of calibration curves for PPD, MAPPD and DAPPD in human blood $(n=3)$ with back calculated concentrations.

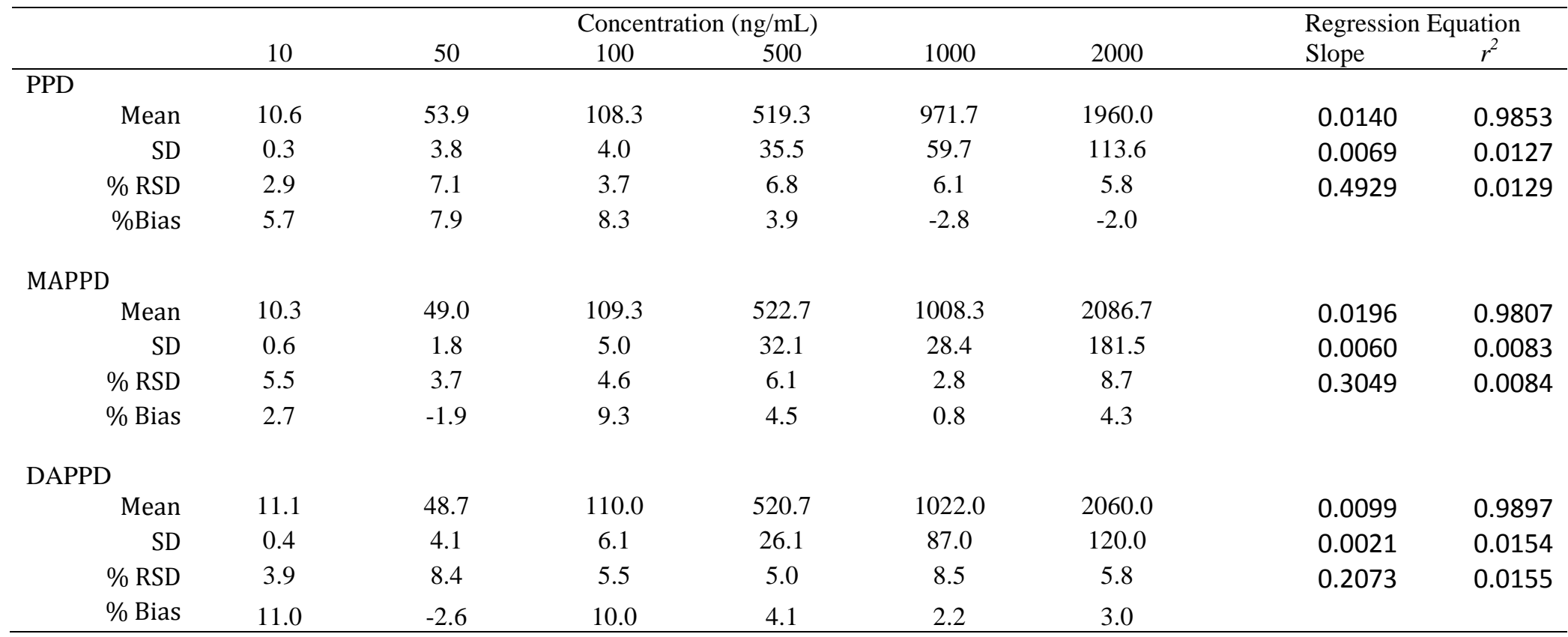

SD: Standard deviation

RSD: Relative standard deviation

$r^{2}$ : Correlation coefficient 


\subsection{Method validation}

\subsubsection{Specificity, sensitivity and linearity}

Six different blank blood specimens were analyzed to evaluate chromatographic interference. Figure $2_{\mathrm{A}}$ and $\mathrm{B}$ represent the total ion chromatogram (TIC) for a blank blood fortified with internal standard, and fortified with LQC. As shown in these figures, no interferences with analytes peaks were detected.

The LOD and LOQ for all analytes were 5 and $10 \mathrm{ng} / \mathrm{mL}$, respectively.

The calibration lines for PPD, MAPPD and DAPPD showed good linearity over the dynamic range 10-2000 ng/mL within three regression curves. Calculated concentrations of each calibrator were compared to target and were within $\pm 20 \%$. An overview of characteristic calibration data over a dynamic range from the LOD/LOQs to $2000 \mathrm{ng} / \mathrm{mL}$ for PPD, MAPPD and DAPPD is presented in Table 2.

\subsubsection{Precision and accuracy and dilution integrity}

Precision and accuracy of the method are presented in Table 3. Intra- and inter-assay imprecisions for all analytes were ranged from 3.67 to $11.18 \%$ and 5.58 to $13.27 \%$ (RSD), respectively. Inter- and inter-assay assay accuracy for all analytes ranged from -14.00 to $5.17 \%$ and -9.47 to $0.86 \%$ (\% bias), respectively.

Dilution accuracy was studied by assaying diluted blood quality control samples. The accuracy for $1 / 10$ dilution samples for all compounds was ranged from -14.00 to $-0.44 \%$.

\subsubsection{Matrix effect and recovery}

With regard to the matrix effect, the comparison between relative standard deviation of the analytes spiked in extracted blank blood samples versus those for pure diluted standards showed less than $10 \%$ analytical signal suppression.

Mean recovery values for PPD, MAPPD and DAPPD were 51.94, 56.20 and 54.88\%, respectively.

\subsubsection{Stability}

Analyte stability evaluations are presented in Table 4. PPD was reduced to $85 \%$ of the original value during the first $6 \mathrm{~h}$ and to $3.1 \%$ after $24 \mathrm{~h}$. The Critical degradation of PPD may be due to 
Table 3

Accuracy and imprecision for the simultaneous determination of PPD, MAPPD and DAPPD in human blood

\begin{tabular}{|c|c|c|c|c|c|c|}
\hline \multirow{2}{*}{$\begin{array}{l}\text { Analyte } \\
\text { (ng/mL) }\end{array}$} & \multicolumn{3}{|c|}{ Intra-assay $(n=6)$} & \multicolumn{3}{|c|}{ Inter-assay $(\mathrm{n}=18)$} \\
\hline & $\begin{array}{c}\text { Mean } \\
(\mathrm{ng} / \mathrm{mL})\end{array}$ & $\begin{array}{c}\text { Accuracy } \\
\text { (\%Bias) }\end{array}$ & $\begin{array}{l}\text { Precision } \\
\text { (\% RSD) }\end{array}$ & $\begin{array}{c}\text { Mean } \\
(\mathrm{ng} / \mathrm{mL})\end{array}$ & $\begin{array}{r}\text { Accuracy } \\
\text { (\% Bias) }\end{array}$ & $\begin{array}{l}\text { Precision } \\
\text { (\% RSD) }\end{array}$ \\
\hline \multicolumn{7}{|l|}{ PPD } \\
\hline 75 & 68.02 & -9.31 & 3.67 & 69.09 & -7.88 & 10.30 \\
\hline 750 & 757.50 & 1.00 & 7.93 & 725.59 & -3.253 & 13.27 \\
\hline 1500 & 1290.00 & -14.00 & 6.58 & 1385.00 & -7.67 & 13.25 \\
\hline \multicolumn{7}{|l|}{ MAPPD } \\
\hline 75 & 65.01 & -13.32 & 11.18 & 69.71 & -7.06 & 12.39 \\
\hline 750 & 688.33 & -8.22 & 5.49 & 734.47 & -2.07 & 9.89 \\
\hline 1500 & 1398.00 & -6.80 & 5.62 & 1358.00 & -9.47 & 5.58 \\
\hline \multicolumn{7}{|l|}{ DAPPD } \\
\hline 75 & 78.88 & 5.17 & 7.60 & 75.65 & 0.86 & 7.77 \\
\hline 750 & 715.75 & -4.57 & 5.95 & 706.88 & -5.75 & 9.03 \\
\hline 1500 & 1498.00 & -0.13 & 3.96 & 1427.75 & -4.82 & 12.72 \\
\hline
\end{tabular}


Table 4

Stability data of PPD, MAPPD and DAPPD in human blood at time interval $(n=4)$.

\begin{tabular}{|c|c|c|c|c|c|c|c|c|c|c|c|}
\hline \multirow[t]{2}{*}{ Analyte } & \multirow[t]{2}{*}{$\begin{array}{c}\text { Target } \\
\text { (ng/mL) }\end{array}$} & \multicolumn{5}{|c|}{$\begin{array}{c}\text { Stability in blood } \\
{\text { Mean concentration (\% of initial concentration })^{\mathrm{b}}}^{\mathrm{a}}\end{array}$} & \multicolumn{5}{|c|}{$\begin{array}{c}\text { Extracts stability } \\
\text { Mean concentration (\% of initial concentration })^{\mathrm{b}}\end{array}$} \\
\hline & & $0 \mathrm{~h}$ & $6 \mathrm{~h}$ & $12 \mathrm{~h}$ & $24 \mathrm{~h}$ & $48 \mathrm{~h}$ & $0 \mathrm{~h}$ & $4 \mathrm{~h}$ & $6 \mathrm{~h}$ & $12 \mathrm{~h}$ & $24 \mathrm{~h}$ \\
\hline \multirow[t]{2}{*}{ PPD } & 75 & $100.0(12.5)$ & $87.7(4.6)$ & $38.2(13.2)$ & $22.1(11.6)$ & $5.5(10.5)$ & $100.0(12.5)$ & $98.1(11.3)$ & $97.5(4.8)$ & $41.3(8.2)$ & $3.1(12.8)$ \\
\hline & 750 & $100.0(16.6)$ & $85.0(12.8)$ & $29.2(14.9)$ & $27.5(8.7)$ & $11.7(14.7)$ & $100.07 .5)$ & $95.6(13.4)$ & $90.1(10.9)$ & $76.0(11.8)$ & $17.4(10.3)$ \\
\hline \multirow[t]{2}{*}{ MAPPD } & 75 & $100.0(12.9)$ & $90.3(14.0)$ & $87.2(11.3)$ & $86.6(1.4)$ & $83.3(11.7)$ & $100.0(13.3)$ & 98.8 (11.9) & $103.0(8.2)$ & $86.0(12.3)$ & $87.0(14.7)$ \\
\hline & 750 & $100.0(8.6)$ & $102.7(4.7)$ & $98.3(9.0)$ & $97.5(12.6)$ & $94.1(13.9)$ & $100.0(5.9)$ & $99.2(4.7)$ & $98.6(4.3)$ & $93.9(15.1)$ & $90.2(14.2)$ \\
\hline \multirow[t]{2}{*}{ DMPPD } & 75 & $100.0(6.9)$ & $108.5(4.0)$ & $111.4(4.4)$ & $99.0(15.0)$ & $98.1(9.4)$ & $100.0(10.0)$ & $111.2(6.3)$ & $103.9(4.0)$ & $105.1(12.2)$ & $110.8(1.8)$ \\
\hline & 750 & $100.0(13.7)$ & $112.6(6.6)$ & $114.3(13.9)$ & $101.5(6.4)$ & $100.8(14.2)$ & $100.0(9.4)$ & $112.6(6.6)$ & $106.3(3.7)$ & $113.9(6.8)$ & $115.9(9.1)$ \\
\hline
\end{tabular}

${ }^{\mathrm{a}}$ Stability at $-20{ }^{0} \mathrm{C}$

${ }^{\mathrm{b}}$ Mean (RSD) 
the autoxidation of PPD by air and/or light [21-22]. Ascorbic acid was used as antioxidant to prevent the oxidation of PPD [18]. In this work, the ascorbic acid was tested to prevent the oxidation of PPD; a significant degradation of PPD was also resulted. PPD was observed to be stable in strong acidic medium or as a hydrochloride salt. To minimize the oxidation of PPD, the samples were extracted in short time and stored in brown vials before the analysis. MAPPD and DAPPD were stable in blood samples and the autosampler up to $48 \mathrm{~h}$ and $24 \mathrm{~h}$, respectively. The RSD for the two quality control samples of MAPPD and DAPPD was within $15 \%$.

\subsection{Application of the method}

The validated method was successfully applied to quantify PPD, MAPPD and DAPPD from poisoning cases by PPD. Figure 2 shows the Total Ion Chromatogram (TIC) of a blood sample spiked with PPD, MAPPD and DAPPD and postmortem blood collected from autopsy cases with suspected PPD poisoning. The concentrations of PPD, MAPPD and DAPPD in antemortem and postmortem blood samples are presented in Table 5.

\section{Table 5}

Concentration levels of PPD, MAPPD and DAPPD $(\mu \mathrm{g} / \mathrm{mL})$ for 8 post-mortem and one antemortem cases.

\begin{tabular}{|c|r|r|r|}
\hline Cases & \multicolumn{1}{l|}{ PPD } & \multicolumn{1}{l|}{ MAPPD } & \multicolumn{1}{l|}{ DAPPD } \\
\hline $\mathbf{1}$ & 3.408 & 3.987 & 1.767 \\
\hline $\mathbf{2}$ & 0.631 & 2.703 & 1.000 \\
\hline $\mathbf{3}$ & 0.287 & 3.015 & 15.076 \\
\hline $\mathbf{4}$ & 0.065 & 2.636 & 14.946 \\
\hline $\mathbf{5}$ & 12.503 & 4.802 & 1.879 \\
\hline $\mathbf{6}$ & 5.876 & 5.316 & 2.140 \\
\hline $\mathbf{7}$ & 0.287 & 6.913 & 11.425 \\
\hline $\mathbf{8}$ & 0.015 & 0.828 & 5.119 \\
\hline $\mathbf{9 *}$ & 0.003 & 0.235 & 2.354 \\
\hline
\end{tabular}

\footnotetext{
* Antemortem case
} 


\section{Conclusion}

To our knowledge, this is the first fully validated LC-MS-MS method for the simultaneous identification and quantification of the PPD and its metabolites MAPPD and DAPPD in human blood. The method was successfully applied to analysis of blood samples collected from poisoning cases by PPD.

\section{Acknowledgments}

This research was supported by the International Federation of Clinical Chemistry and Laboratory Medicine (IFCC). The authors would like to acknowledge the Department of Pharmacology, University of Pretoria and Assiut Chemical Laboratory of Egyptian MedicoLegal Department for providing necessary facilities to carry out this work. We would also like to thank all staff of Clinical Toxicology Laboratory of Faculty of Medicine, Sohag University, Egypt for providing biological samples.

\section{References}

[1] W. Ashraf, S. Dawling, L.J. Farrow, Hum Exp Toxicol 13 (1994) 167-170.

[2] K.S. Chugh, G.H. Malik, P.C. Singhal, J. Med 13 (1982)131-137.

[3] A.A. Zeggwagh, R. Aboukal, R. Madani, A. Zerkaoui, O. Kerkeb, Ann Fr Anesth Reanim 19 (2003) 639-641.

[4] S. Kumar, J Indian Acad Forensic Med 32 (2010) 163-164.

[5] S.A. Shalaby, M.K. Elmasry, A.E. Abd-Elrahman, M.A. Abd-Elkarim, Z.A. Abd- Elhaleem, Toxicol Ind Health 26 (2010) 81-87.

[6] A. Chrispal, A. Begum, I. Ramya, A. Zachariah, Trop Doct 40 (2010) 100103.

[7] K.M. Mohamed M.A. Hilal, N.S. Aly, IJFP 2:301 (2014) 1-5

[8] N. Takahashi, J. Ishizawa, M. Yamashita, Jpn J Acute Med 12 (1988) 1509-1512.

[9] J. Ishizawa, Takahashi N., Jpn J Acute Med 20 (1996) 1584-1585.

[10] H. Shima, Med J Nihon Univ 19 (1960) 2633-2638.

[11] M. Nakao, Y. Takeda, Yakugaku Zasshi 99 (1979) 1149-1153.

[12] N. Goetz, P. Laserre, P. Bore, G. Kalopissis, Int. J. Cosmet. Sci. 10 (1988) 63-73. 
[13] Y. Kawakubo, H.F. Merk, T.A. Masaoudi, S, Sieben, B. Blomeke, J Pharmacol Exp Ther 292 (2000) 150-155.

[14] K. Koyama, N. Ogasawara, K. Kadomatsu, IRYO 54 (2000) 565-570.

[15] A. Stambouli, M.A. Bellimam, N. El-Karni, T. Bouayoun, A. El-Bouri, Forensic Sci. Int. 146S (2004) S87-S92.

[16] L. Wang, S. Tsai, Anal. Biochem. 312 (2003) 201-207.

[17] G.P. Hooff, N.A. van Huizen, R.J.W. Meesters, E.E. Zijlstra, M. Abdelraheem, PLoS ONE 6 (2011) 1-8.

[18] A. Meyer, B. Blömekeb, K. Fischer, J. Chromatogr. B 877 (2009) 1627-1633.

[19] Guidance for Industry: Bioanalytical Method Validation, U.S. Department of Health and Human Services, Food and Drug Administration Centre for Drug Evaluation and Research (CDER), Centre for Veterinary Medicine (CVM), May 2001.

[20] B.K. Matuszewski, M.L. Constanzer, C.M. Chavez-Eng, Anal Chem 75 (2003) 3019-3030.

[21] J.F. CORBETT, J. Sot. Cosmet. Chem. 23 (1972) 683-93.

[22] S. Jahn, H.Faber, R. Zazzeroni, U. Karst, Rapid Commu. Mass Spectrom. 26 (2012) 14531464. 